\title{
Performance analysis of IEEE 802.11 ac wireless backhaul networks in saturated conditions
}

\author{
Ruizhi Liao, Boris Bellalta*, Jaume Barcelo, Victor Valls and Miquel Oliver
}

\begin{abstract}
According to the ongoing IEEE 802.11 ac amendment, the wireless network is about to embrace the gigabit-per-second raw data rate. Compared with previous IEEE standards, this significant performance improvement can be attributed to the novel physical and medium access control (MAC) features, such as multi-user multiple-input multiple-output transmissions, the frame aggregation, and the channel bonding. In this paper, we first briefly survey the main features of IEEE 802.11ac, and then, we evaluate these new features in a fully connected wireless mesh network using an analytic model and simulations. More specifically, the performance of the MAC scheme defined by IEEE 802.11ac, which employs the explicit compressed feedback (ECFB) mechanism for the channel sounding, is evaluated. In addition, we propose an extended request-to-send/clear-to-send scheme that integrates the ECFB operation to compare with the IEEE 802.11ac-defined one in saturated conditions. The comparison of the two MAC schemes is conducted through three spatial stream allocation algorithms. A simple but accurate analytical model is derived for the two MAC schemes, the results of which are validated with simulations. The observations of the results not only reveal the importance of spatial stream allocations but also provide insight into how the newly introduced features could affect the performance of IEEE 802.11ac-based wireless mesh networks.
\end{abstract}

Keywords: IEEE 802.11ac; Wireless mesh backhaul networks; MAC; MU-MIMO; Saturation throughput

\section{Introduction}

IEEE 802.11 [1] is the de facto standard of the widely deployed wireless local area networks (WLANs). Since its debut in 1997, it comes a way from megabits per second to the upcoming gigabits per second [2], which was achieved by the cable technology not long ago. The currently ongoing IEEE 802.11ac amendment [3] aims to provide an aggregated multi-station throughput of at least 1 gigabit per second in the $5-\mathrm{GHz}$ band. This performance improvement, compared to IEEE 802.11n [4], is obtained by introducing novel physical layer (PHY) and medium access control layer (MAC) features, including (1) wider channel bandwidths, (2) a higher modulation scheme, (3) downlink multi-user multiple-input multipleoutput (MU-MIMO) transmissions, and (4) a compulsory frame aggregation mechanism. IEEE 802.11ac also introduces other novel features, e.g., the TXOP Sharing, while we only focus on those tightly related to this paper.

*Correspondence: boris.bellalta@upf.edu

NeTS Research Group, Department of Information and Communication Technologies, Universitat Pompeu Fabra, Barcelona 08018, Spain
Some previous work has investigated the performance of IEEE 802.11ac focusing on one of the features. In [5], Redieteab et al. utilize a PHY and MAC cross-layer simulation platform to explore the impact of the training interval on the system performance. The results show that a frequent training process helps to increase the throughput in spite of the associated overhead. In [6], Cha et al. compare the performance of a downlink MUMIMO scheme with a space-time block coding-based frame aggregation scheme. The authors claim that the MU-MIMO scheme produces a higher throughput than the other if transmitted frames are of similar length. In [7], Ong et al. compare the MAC throughput of IEEE 802.11ac with that of IEEE 802.11n over different frame aggregation schemes. The results suggest that a hybrid scheme of aggregated MAC protocol data unit (A-MPDU) and aggregated MAC service data unit (A-MSDU) yields the best performance. In [8], Bellalta et al. present a frame aggregation scheme for IEEE 802.11ac WLANs and evaluate its performance in non-saturated conditions. The results show that both the number of active nodes and

\section{Springer}

C 2013 Liao et al : licensee Springer. This is an Open Access article distributed under the terms of the Creative Commons Attribution License (http://creativecommons.org/licenses/by/2.0), which permits unrestricted use, distribution, and reproduction in any medium, provided the original work is properly cited. 
the queueing length have significant impacts on the system performance. In [9], Nojima et al. measure the performance of a MU-MIMO WLAN system in a realistic channel, where two linear precoding schemes, channel inversion (CI) and block diagonalization (BD), are applied and compared. From the results, the authors conclude that the $\mathrm{BD}$ precoding scheme is more effective than the CI.

Bianchi in [10] considers a legacy IEEE 802.11 network using the distributed coordination function and derives the prominent saturation throughput analytical model for both basic access and request-to-send/clearto-send (RTS/CTS) schemes. This saturation throughput analytic model is extended to support the MU-MIMO transmission and conformed to IEEE 802.11ac parameters in this paper. Regarding MU-MIMO transmissions in WLANs, Li et al. propose an integrated MU-MAC protocol that includes both multi-packet transmission (MPT) and multi-packet reception (MPR) in [11], where the perfect channel state information (CSI) is assumed to be obtained by the RTS/CTS training. The authors claim that the integration of MPT and MPR can obtain a significant performance improvement in terms of the number of supported nodes. Some related performance evaluation on MPT, MPR, or a combination of MPT and MPR schemes for wireless networks can be found in [12-14].

In this paper, we capture the most important features of IEEE 802.11ac (e.g., MU-MIMO, the channel sounding interval, the number of antennas, the size of aggregated frames, and the channel bandwidth) to get insight into how these parameters can affect the system performance. The considered scenario is a fully connected wireless mesh network (fully connected means that all nodes are directly connected), which is targeted by the IEEE 802.11ac usage models [15]. As can be seen in Figure 1, mesh nodes are equipped with two interfaces: an IEEE 802.11ac, which is used to communicate with mesh nodes, and an IEEE $802.11 \mathrm{n}$, which is used to communicate with the associated stations of each WLAN. In this paper,

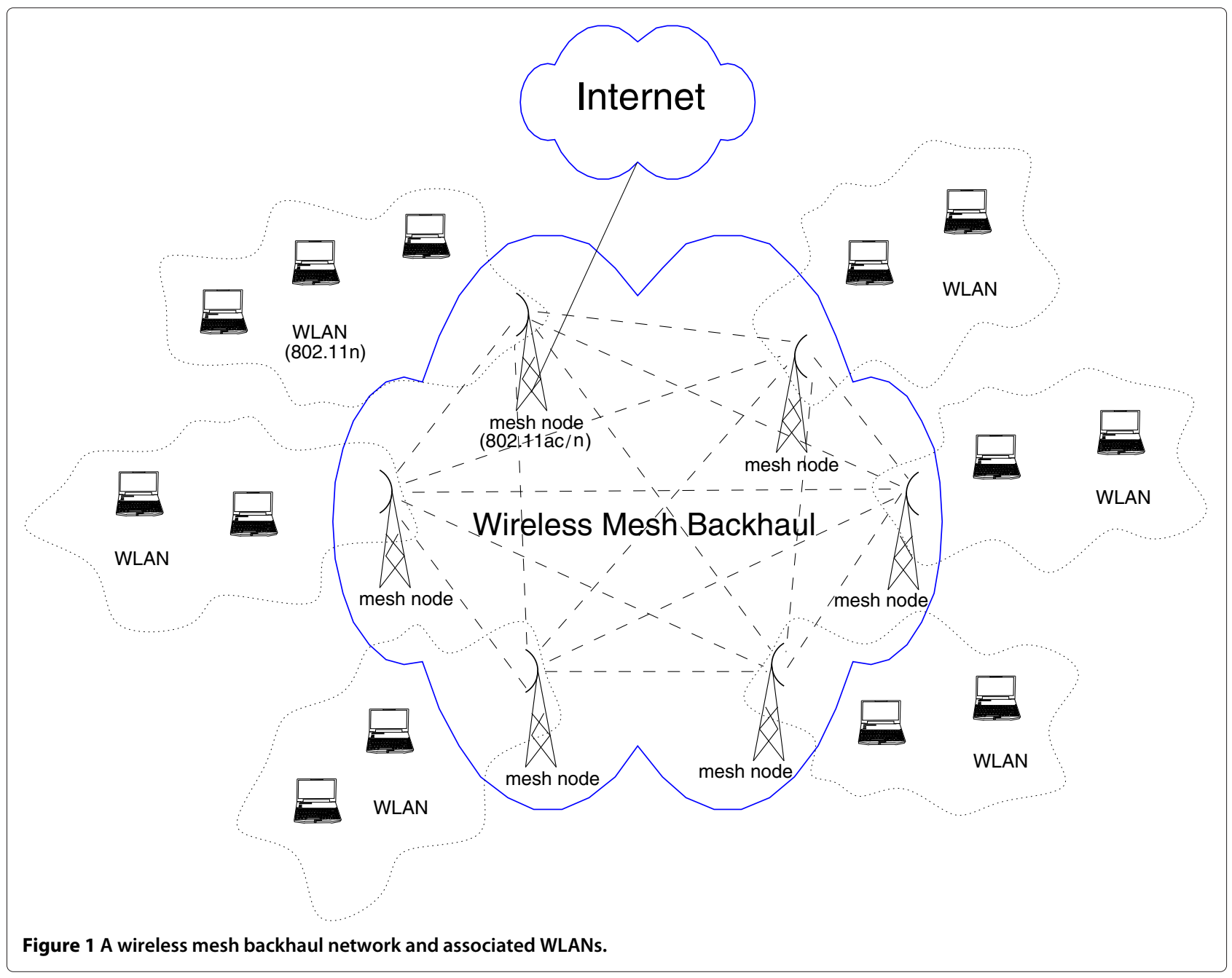


we focus on the performance analysis of the wireless mesh backhaul network, assuming that all the mesh nodes have the same number of antennas, which enables the transmission of multiple frames to the same or different destinations.

The contributions of the paper are the following: (1) A brief survey of the novel IEEE 802.11ac features that are closely related to the performance analysis of this paper is presented. (2) The procedure of the IEEE 802.11ac-defined basic access scheme is illustrated. For comparison purposes, an extended RTS/CTS scheme that integrates the channel sounding protocol to support the MU-MIMO transmission is proposed. (3) A saturation throughput analytic model for both schemes is derived, the results of which are verified with those of the simulation. (4) Three spatial stream allocation algorithms are designed to compare the performance of the two MAC schemes by increasing the number of nodes, the number of antennas, the size of A-MPDU, and the channel bandwidth, with special attention being paid to the impact of the training process on the system performance.

In what follows, the basic access scheme defined by IEEE 802.11ac and the extended RTS/CTS scheme proposed by us are named as MU-Basic and MU-RTS/CTS, respectively. The rest of the paper is organized as follows. First, Section 2 surveys the main characteristics of IEEE 802.11ac. Then, Section 3 explains how the MU-Basic and the MU-RTS/CTS protocols work. After that, Section 4 presents the spatial stream allocation algorithms, the analytic model, and the simulation results. Finally, Section 5 concludes the paper and looks into the open research challenges.

\section{IEEE 802.11ac}

This section briefly surveys the upcoming amendment IEEE 802.11ac, including the PHY layer and the MAC layer enhancements. This review does not go through every aspect of IEEE 802.11ac but focuses on those techniques and parameters which are needed to understand how the IEEE 802.11ac-based protocols work and how they could affect the system performance. A summary of IEEE 802.11ac main characteristics is shown in Table 1.

\subsection{IEEE 802.11 ac main features \\ 2.1.1 Wider channel bandwidth}

Compared to the legacy standard, IEEE 802.11ac operates exclusively in the 5-GHz band, which avoids interferences from many legacy devices as well as household appliances that operate at $2.4 \mathrm{GHz}$. In addition, there are more nonoverlapping channels at $5 \mathrm{GHz}$, which can be bonded together to obtain wider channels. IEEE 802.11ac adds $80-$ and $160-\mathrm{MHz}$ (optional) channels into its specification, where the $80-\mathrm{MHz}$ channel is formed by combining
Table 1 IEEE 802.11ac parameters

\begin{tabular}{ll}
\hline Parameters & Values \\
\hline $\begin{array}{l}\text { Spectrum } \\
\text { Max number of simultaneous }\end{array}$ & $5 \mathrm{GHz}$ \\
receiving nodes & 4 \\
$\begin{array}{l}\text { Max number of simultaneous } \\
\text { streams to a node/all nodes }\end{array}$ & $4 / 8$ \\
Aggregation scheme & A-MPDU, A-MPDU of A-MSDU \\
Maximum A-MPDU size & $1,048,575$ bytes \\
$\begin{array}{l}\text { Channel sounding } \\
\text { (CSI feedback) }\end{array}$ & ECFB training protocol (optional) \\
Modulation schemes & BPSK, QPSK, 16-QAM, 64-QAM, \\
Channel bandwidth & $256-\mathrm{QAM}$ (optional) \\
& 20,40, and 80 MHz, \\
Guard interval & 160 and $80+80$ MHz (optional) \\
& $0.8 \mu$ s, \\
Forward error correction & $0.4 \mu$ s (optional) \\
& Binary convolutional coding, \\
Bandwidth indication & Low-density parity check (optional) \\
& CTS, \\
\hline
\end{tabular}

two adjacent $40-\mathrm{MHz}$ channels, and the $160-\mathrm{MHz}$ channel is built up by combining two adjacent or non-adjacent 80-MHz channels [16].

\subsubsection{Higher modulation and coding scheme}

The 64-quadrature amplitude modulation (QAM) with $5 / 6$ coding rate is the highest modulation and coding scheme (MCS) employed in IEEE 802.11n, which is extended to 256-QAM in IEEE 802.11ac. With 256-QAM, each symbol can carry eight information bits, increasing the number of transmitted bits per hertz. However, 256-QAM requires a higher signal-to-noise ratio at the receiving end in order to keep a low-bit error probability compared with other modulation schemes included in IEEE 802.11ac [17].

\subsubsection{CSI Feedback for multi-user beamforming}

IEEE 802.11ac utilizes a channel sounding protocol called explicit compressed feedback (ECFB, as shown in Figure 2) to obtain the required CSI for multi-user beamforming $[16,18]$. The ECFB protocol works as follows. The beamformer first sends a null data packet announcement (NDPA) to initiate the training process, which includes the addresses of the targeted nodes. After a short interframe space (SIFS) interval, the beamformer will send a null data packet (NDP), where a set of training sequences that are known by both the beamformer and the beamformees will be included into the very high throughput long training field (VHT-LTF). The node that is identified as the 


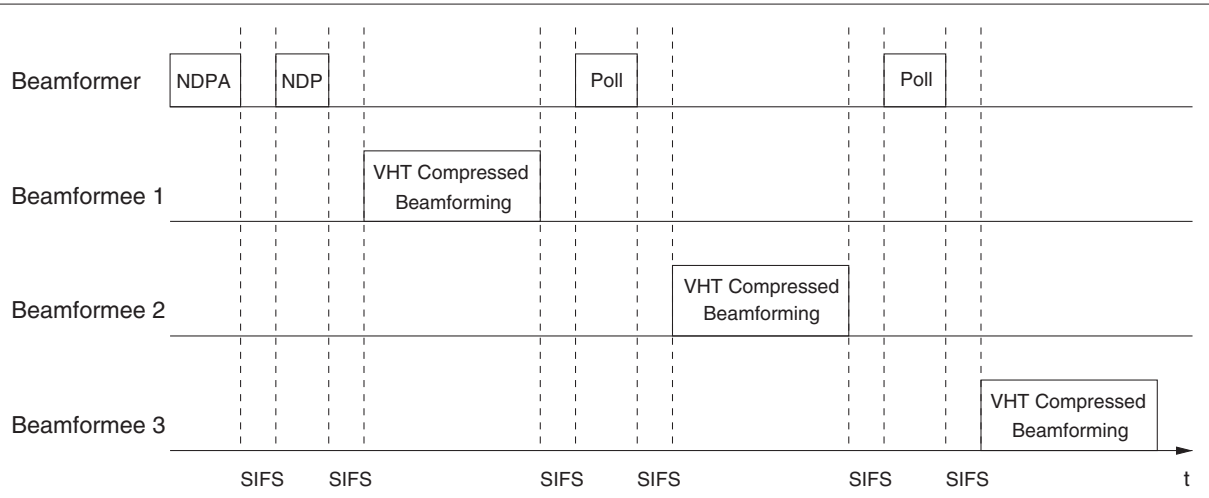

Figure 2 An example of the ECFB of three beamformees.

first responder in the NDPA utilizes the training sequence in the NDP to estimate the channel and compresses the measured channel information, which is then fed back in a VHT compressed beamforming frame. The volume of the CSI feedback depends on the number of antennas and sub-carriers. Other nodes who are addressed in the NDPA will respond if they are explicitly polled. With these replied VHT compressed beamforming frames, the beamformer can calculate the weight and precisely steer each beam to the targeted receiver. For the detailed structure of NDPA, NDP, VHT compressed beamforming frame, and Poll, readers can refer to $[3,4]$.

\subsubsection{Spatial multiplexing}

A maximum number of four spatial streams is defined in IEEE $802.11 \mathrm{n}$ for the point-to-point communication mode (single-user MIMO). IEEE 802.11ac extends this maximum number from four to eight. In order to restrict the MU-MIMO transmission to a manageable scale, IEEE 802.11ac specifies the two following rules to the eight spatial streams: (1) The maximum number of simultaneous beams directed to different nodes is four, which means that the maximum number of simultaneous receivers of a MU-MIMO transmission is four. (2) The maximum number of simultaneous spatial streams inside a beam towards a node is four, which means that the maximum number of spatial streams that each receiver can have is also four $[16,17]$.

\subsection{IEEE 802.11ac PHY frame format}

Figure 3 shows the structure of the IEEE 802.11ac PHY frame, where PLCP and PPDU stand for physical layer convergence protocol and PLCP protocol data unit, respectively. A service field and a tail field are appended to the A-MPDU before being processed by the PHY layer. The PHY header starts with a preamble that includes three legacy fields [3]: the legacy short training field (L-STF), the legacy long training field (L-LTF), and the legacy signal field (L-SIG). L-STF and L-LTF have functions such as detecting signal, synchronization, and frequency offset estimation. L-SIG contains information about the data rate and the length of the transmitted frame. These legacy fields are kept for the backward compatibility.

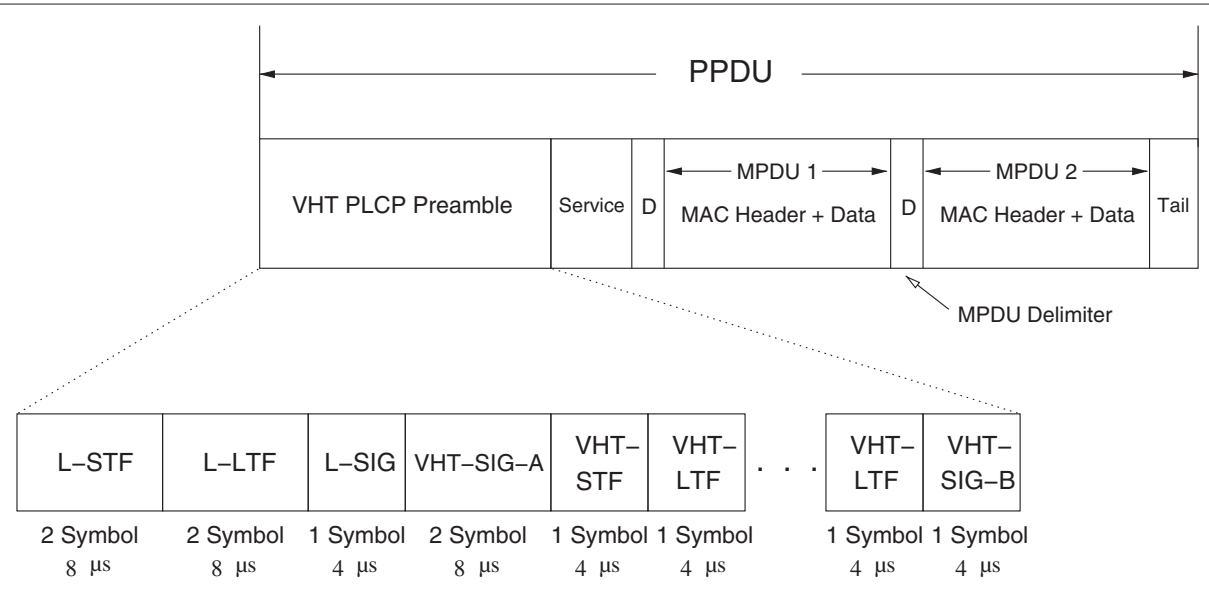

Figure 3 PHY frame format of IEEE 802.11 ac. 
The following VHT fields are introduced by IEEE 802.11ac to assist the novel PHY and MAC features in obtaining the required information [3,7]. VHT-SIG-A consists of two orthogonal frequency division multiplexing (OFDM) symbols: VHT-SIG-A1 and VHT-SIG-A2, each containing 24 bits. Note that there is a group identifier (Group-ID) field in VHT-SIG-A1, which is utilized to notify those nodes about the following MU-MIMO transmission. VHT-STF is a short training field that is used to compute the transmission power assigned to that transmission. VHT-LTF allows a receiver to estimate the MIMO channel by containing a training sequence that is known by both the transmitting and the receiving nodes. IEEE 802.11ac supports up to eight VHT-LTF fields, each of which contains an orthogonal training sequence. In order to precisely estimate the MIMO channel, the number of VHT-LTF fields should be equal to or higher than the number of transmitted spatial streams. The VHT-SIG$B$ field contains the length of data and the MCS used for each beam.

\subsection{IEEE 802.11ac MAC enhancements}

\subsubsection{A-MPDU}

IEEE 802.11n introduces the A-MSDU and the A-MPDU frame aggregation schemes to improve the MAC efficiency. MSDUs who share a common MAC header are aggregated into an A-MSDU, which is then encapsulated into an MPDU. Multiple MPDUs with different MAC headers are aggregated into an A-MPDU. In IEEE 802.11ac, the maximum size of A-MSDU and A-MPDU has been increased to 11,406 and $1,048,575$ bytes, respectively, and all frames are required to be transmitted as the format of A-MPDU even if there is only one MPDU $[3,16]$.

\subsubsection{Group-ID}

Group-ID is a field defined in VHT-SIG-A1 to signal a group of selected receivers. More specifically, the GroupID field is utilized by a receiving node to decide if it is targeted in the followed MU-MIMO transmission. Depending on whether a node is targeted, it will either check the user position field (a field defined in VHT-SIGA1) to identify the spatial streams that correspond to itself or will not process the rest of the PPDU [3]. Although Group-ID is specified in the frame of the PHY layer, it actually benefits the MAC layer because the control frame (e.g., RTS) or the MAC header of data frames does not need to be extended to accommodate multiple receivers' addresses.

\subsection{IEEE 802.11ac data rate}

In IEEE $802.11 \mathrm{ac}$, the data rate is $R_{\text {data }}=\frac{N_{\text {DBPS }}}{T_{\text {symbol }}}$, where $N_{\text {DBPS }}$ is the number of data bits per OFDM symbol and $T_{\text {symbol }}$ is the symbol duration. $N_{\text {DBPS }}$ is determined by the number of data sub-carriers $\left(N_{\mathrm{dsc}}\right)$ and MCS, while
$T_{\text {symbol }}$ is determined by the employed bandwidth and the guard interval (GI).

An example to calculate the maximum data rate of a single spatial stream is shown as follows. In a scenario where the channel bandwidth is $160 \mathrm{MHz}$ and GI is $0.4 \mu \mathrm{s}$, there will be 468 data sub-carriers out of 512 OFDM subcarriers; each OFDM symbol of a sub-carrier can carry up to $8 \cdot 5 / 6$ information bits if a 256-QAM with 5/6 coding rate is employed. The total number of data bits in a symbol, $N_{\text {DBPS }}$, equals $468 \cdot 8 \cdot 5 / 6=3,120 \mathrm{bits} /$ symbol. The symbol duration, $T_{\text {symbol }}$, is equal to $512 / 160 \mu \mathrm{s}+0.4$ $\mu \mathrm{s}=3.6 \mu \mathrm{s}$. Then the maximum single-stream data rate is obtained: $\frac{N_{\text {DBPS }}}{T_{\text {symbol }}} \approx 866.7 \mathrm{Mbps}$.

\section{MU-MIMO MAC protocols for IEEE 802.11 ac}

In this section, the IEEE 802.11ac-defined basic access scheme (MU-Basic) and the proposed one (MURTS/CTS) are introduced, both of which are based on the IEEE 802.11 enhanced distributed channel access (EDCA).

\subsection{MU-Basic}

A node running in the MU-Basic scheme switches between two modes: the ECFB training (CSI mode) and the multi-user data transmission (data mode). The ECFB training is periodically $\left(T_{2-\mathrm{CSI}-\mathrm{Req}}\right)$ performed by each node to obtain the required CSI from its neighbors. $T_{2-\text { CSI-Req }}$ is the interval between two CSI requests of a node. The operations of ECFB have been described in Section 2.1. This section focuses on the multi-user data transmission.

The MU-Basic scheme is based on EDCA, with the difference that a node who wins the channel is able to send frames to multiple receivers. After the channel has been idle for an arbitration inter frame space (AIFS), a back-off (BO) starts to count down. As soon as a node's BO first reaches zero, it simultaneously transmits multiple A-MPDUs. If all frames are successfully received, the receiving nodes will send block $\mathrm{ACKs}$ (B-ACKs) sequentially. Note that a node has a unique BO no matter whether it is in the CSI mode or in the data mode, which is to say that if the node switches to the CSI mode in the middle of the data mode's $\mathrm{BO}$ countdown process, it will utilize the ongoing $\mathrm{BO}$ for the ECFB frames.

In this paper, the bitmap field of B-ACK is set to be a variable to account for the number of aggregated MPDUs $\left(N_{\mathrm{f}}\right)$ in each A-MPDU. The frame fields of B-ACK are shown in Table 2.

An example of a successful MU-Basic transmission is illustrated in Figure 4. Initially the channel is assumed busy (B) in Figures 4, 5, and 6. Suppose node 1 wins the channel contention and simultaneously transmits two AMPDUs, one is directed to node 2 and the other is directed 
Table 2 Frame fields of B-ACK

\begin{tabular}{|c|c|c|c|c|c|c|c|}
\hline 2 bytes & 2 bytes & 6 bytes & 6 bytes & 2 bytes & 2 bytes & {$\left[N_{f} / 8\right]$ bytes } & 4 bytes \\
\hline Frame & Duration & Receiver & Transmitter & B-ACK & Starting & B-ACK & Frame \\
\hline \multirow[t]{2}{*}{ control } & & address & address & control & sequence & bitmap & check \\
\hline & & & & & control & & sequence \\
\hline
\end{tabular}

to node 4. After the successful reception of the A-MPDUs, nodes 2 and 4 send the B-ACK sequentially.

If collisions occur, as illustrated in Figure 5, no frames can be received successfully, where the dashed frames mean that these frames would be there if the transmission was successful (no collisions). ECFB-EIFS and MUBasicEIFS represent the extended inter frame space (EIFS) for each case, examples of the calculation of which can be found in $[19,20]$.

\subsection{MU-RTS/CTS}

The MU-RTS/CTS scheme is proposed as an alternative to MU-Basic, where the ECFB training protocol is integrated into the RTS and MU-CTS handshaking process. More specifically, the training sequence is included into the VHT-LTF field of the RTS control frame. The nodes who are targeted in the Group-ID field will estimate the channel and feed back the measured CSI through MUCTSs to the RTS sender. The frame fields of MU-CTS are shown in Table 3. The channel information field contains the measured CSI, which has the same size as that of the VHT compressed beamforming frame, and is equal to $M \cdot N_{\mathrm{dsc}} \cdot 8$ bits, where $M$ is the number of antennas of each node, $N_{\mathrm{dsc}}$ is the number of data sub-carriers, and 8 is the number of bits required to estimate the channel gain of each data sub-carrier.

The benefits of employing the MU-RTS/CTS scheme are as follows: (1) It eliminates the need to periodically execute the ECFB protocol. (2) It reduces the collision time because the length of RTS is much shorter than that

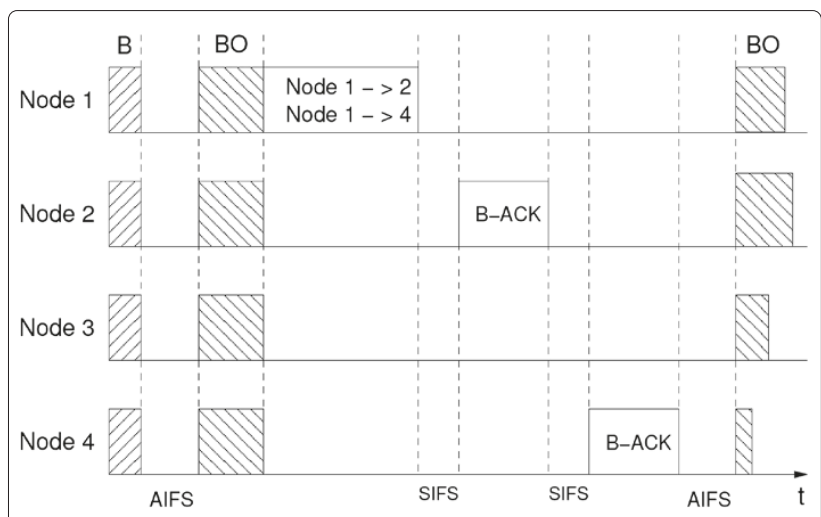

Figure 4 A successful transmission of the MU-Basic scheme. of A-MPDU. (3) The data sender can also obtain CSI by estimating the training sequence included in MU-CTSs, which enables it to receive B-ACKs in parallel, therefore further reducing temporal overheads.

The MU-RTS/CTS scheme works as illustrated in Figure 6a. Suppose that node 1 initiates the transmission by sending an RTS, in which the targeted nodes are mapped in the Group-ID field, and the training sequence is added in the VHT-LTF field of the PHY preamble. The targeted nodes will estimate the channel, include the measured CSI into the channel information field of MU-CTS, and send it back in the same order as indicated in the Group-ID field. With these MU-CTSs that include the required CSI, node 1 is able to create multiple beams towards the selected destinations. In order to take benefits of receiving multiple B-ACKs in parallel, node 1 will also measure the channel from the training sequence of the MU-CTS's VHT-LTF field. If collisions happen, as shown in Figure 6b, MU-RTS/CTS-EIFS, which is set according to the MU-CTS timer, will make all nodes recover from collisions at the same time $[19,20]$.

\section{Saturation throughput analysis and simulation results}

The considered wireless mesh backhaul network is shown in Figure 7. There are $n$ identical mesh nodes, each of which is equipped with $M$ antennas. All these nodes are within the transmitting range of each other, hence forming a fully connected mesh network. A single transmission rate is used for both control frames and data frames. An error-free channel is considered.

All nodes are saturated and transmit fixed length frames of $L$ bits. Frames destined to the same node are assembled into an A-MPDU, which will be assigned to a beam. Each beam contains one or more spatial streams. The AMPDU payload is served by the spatial streams of a beam; therefore, the transmission duration of a beam with more spatial streams is shorter than that of a beam with fewer spatial streams. In order to make all beams of a transmission have the same duration, we assume that a node assigns the same number of spatial streams to different beams in each transmission.

\subsection{Spatial stream allocation algorithms}

Three spatial stream allocation schemes are designed to investigate whether it is better to employ more beams or 


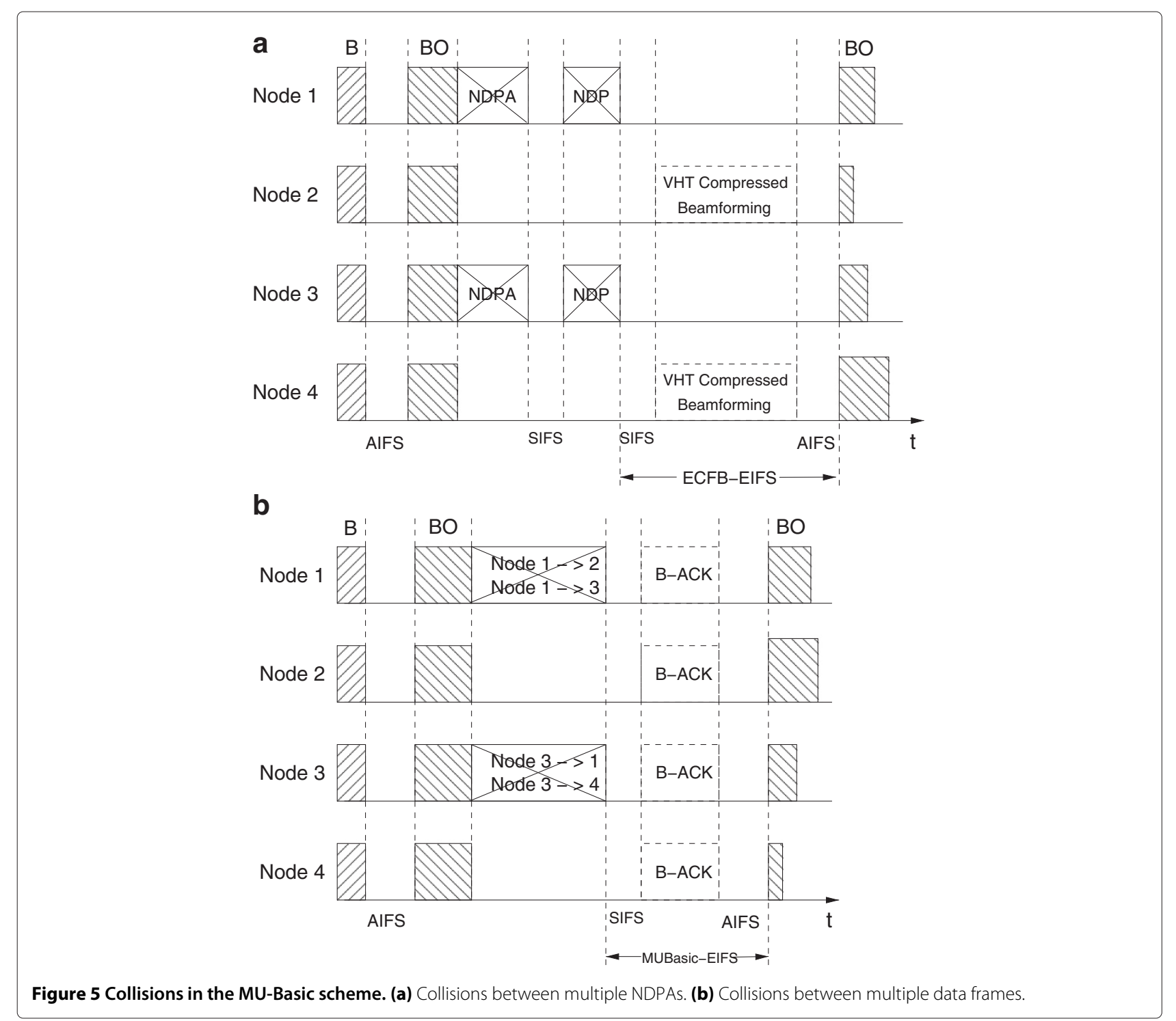

more spatial streams in MU-MIMO transmissions. The algorithms seek the maximum product of the number of beams $\left(N_{\mathrm{b}}\right)$ and the number of spatial streams $\left(N_{\mathrm{s}}\right)$, which can be formulated as Equation 1. $N_{\mathrm{s}, i}=N_{\mathrm{s}, j}$ is the constraint that all beams have to contain the same number of spatial streams, where $i$ and $j$ refer to any pair of beams.

$$
\begin{array}{ll}
\text { Maximize } & N_{\mathrm{b}} \cdot N_{\mathrm{s}} \\
\text { Subject to } & N_{\mathrm{b}} \leq \min (M, \min (n-1,4)) \\
& N_{\mathrm{s}} \leq \min (M, 4) \\
& N_{\mathrm{b}} \cdot N_{\mathrm{s}} \leq \min (M, 8) \\
& N_{\mathrm{s}, i}=N_{\mathrm{s}, j}
\end{array}
$$

The three algorithms are as follows:

1. Stream-greedy algorithm. It tries to maximize the number of spatial streams assigned to a beam, taking into account that the maximum number is 4 . The duration of a transmission is reduced if more spatial streams are assigned to a beam. Therefore, nodes will be able to transmit more frequently, which is expected to improve the network performance. Note that this scheme only parallelizes the payload of A-MPDU over the spatial streams, but not the protocol overheads, such as control frames (e.g., RTS and B-ACK) and the PHY header.

2. Beam-greedy algorithm. It tries to maximize the number of parallel beams, taking into account that the maximum number is 4 . This scheme tries to increase the number of nodes that can simultaneously receive A-MPDUs, which makes the transmission more efficient as the PHY headers are also transmitted in parallel. However, the channel will remain busy for a longer period because the duration of each transmission is 

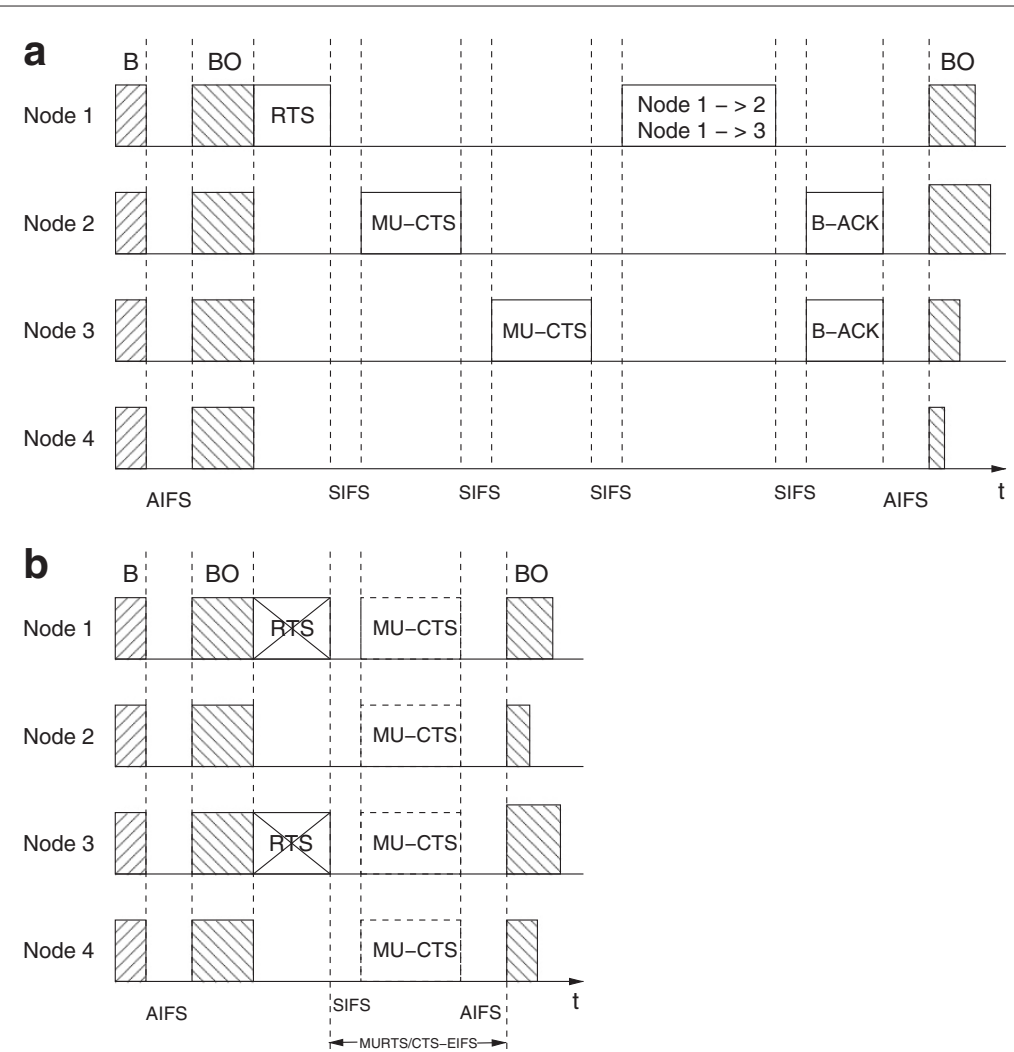

Figure 6 The MU-RTS/CTS scheme. (a) A successful transmission of the MU-RTS/CTS scheme. (b) Collisions between RTSs.

longer, thus reducing the frequency that nodes can transmit new frames.

3. Stream-independent algorithm. It considers that each spatial stream is independent and responsible for transmitting an A-MPDU, regardless of whether AMPDUs are destined to different nodes or to the same node. Therefore, $N_{\mathrm{b}} \leq \min (M, \min (n-1,4))$ reduces to $N_{\mathrm{b}} \leq \min (M, n-1)$. This ideal scheduler aims to further extend the advantages of the Beam-greedy algorithm by removing the limitation on the number of beams that can be simultaneously transmitted.

An example to obtain the solution for each algorithm is as follows. In a scenario where $M=6$ and $n=8$, the maximum value of $N_{\mathrm{b}} \cdot N_{\mathrm{s}}$ is 6 according to Equation 1 . Three solutions are therefore obtained: solution $1, N_{\mathrm{b}}=2$, $N_{\mathrm{s}}=3$; solution $2, N_{\mathrm{b}}=3, N_{\mathrm{s}}=2$; and solution $3, N_{\mathrm{b}}=6$, $N_{\mathrm{s}}=1$. Based on the characteristics of the scheduling

Table 3 Frame fields of MU-CTS

\begin{tabular}{ccccc}
\hline $\mathbf{2}$ bytes & $\mathbf{2}$ bytes & $\mathbf{6}$ bytes & $\boldsymbol{M} \cdot \boldsymbol{N}_{\text {dsc }}$ bytes & $\mathbf{4}$ bytes \\
\hline Frame & Duration & Receiver & Channel & Frame check \\
control & & address & information & sequence \\
\hline
\end{tabular}

algorithms, each one chooses a solution. In other words, the Stream-greedy chooses solution 1, the Beam-greedy takes solution 2, while the Stream-independent goes for solution 3 .

\subsection{Saturation throughput analysis}

In order to simplify the analysis for the MU-Basic scheme, we assume that a slot is previously assigned to either the ECFB training part or the data transmission. Therefore, only collisions among data transmissions or collisions among NDPA frames are counted. $\gamma$ is used to refer to the probability that one slot is allocated for ECFB, and $1-\gamma$ to refer to the probability that one slot is allocated for data transmissions.

Regardless of whether the MU-Basic scheme or the MU-RTS/CTS scheme is used, nodes will start a random back-off counter to compete for accessing the channel if it has been idle for AIFS. Then, each node decreases its random back-off counter by one if the channel is detected as free; otherwise, it freezes the counter. In the latter case, the frozen counter will be reused in the next channel access contention. That is to say, in between each decrement of the back-off counter, a node could observe the channel in either an empty state (no transmission activities) or a busy state (a successful 


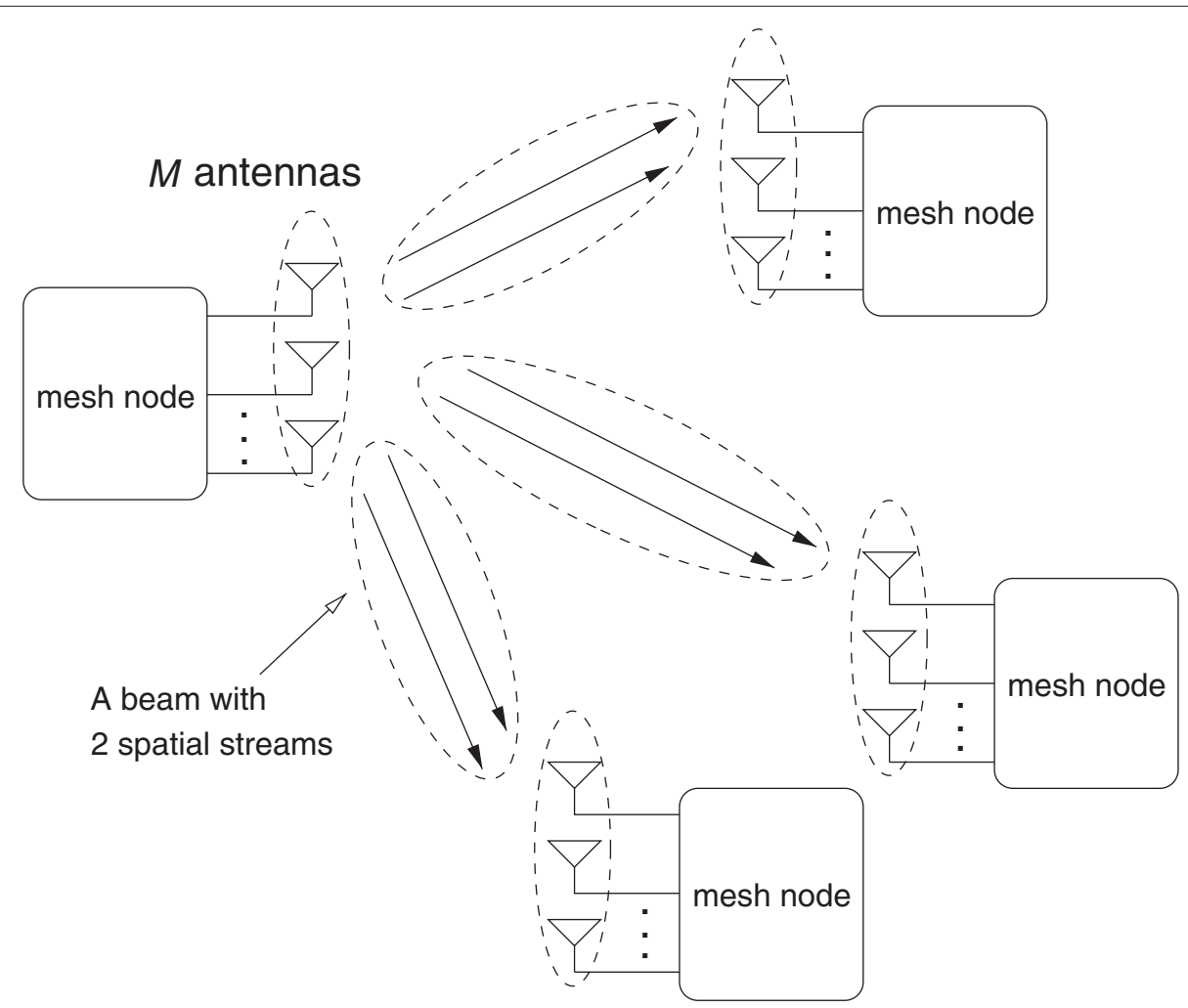

Figure 7 A wireless mesh backhaul network.

transmission or collisions). Therefore, a slot time could be a constant value $\sigma$ if the channel is empty, $T_{\mathrm{s}}$ if there is a successful transmission, or $T_{\mathrm{c}}$ if there are collisions.

Let the transmission probability of each node in a randomly chosen slot be $\tau$. Then, the probability that the channel is empty, $p_{\mathrm{e}}$, is given in Equation 2, which is the probability that no node transmits in that slot.

$$
p_{\mathrm{e}}=(1-\tau)^{n}
$$

The probability that a slot contains a successful transmission, $p_{\mathrm{s}}$, is based on the fact that there are $n$ nodes and only one single node transmits.

$$
p_{\mathrm{S}}=\left(\begin{array}{l}
n \\
1
\end{array}\right) \tau(1-\tau)^{n-1}=n \tau(1-\tau)^{n-1}
$$

Thus, the collision probability in a slot, $p_{\mathrm{c}}$, is obtained as follows:

$$
p_{\mathrm{c}}=1-p_{\mathrm{e}}-p_{\mathrm{s}}
$$

The saturation throughput $S$, as shown in Equation 5, is expressed as the ratio of the frame payload successfully transmitted in a slot and the average duration of a slot, where $\gamma$ is the probability that a slot is labeled as ECFB,
$N_{\mathrm{f}}$ is the number of frames in each A-MPDU, and $N_{\mathrm{b}}$ is the number of parallel beams towards multiple nodes. $L$ is the length of a data frame. $T_{\text {data,s }}$ and $T_{\text {data,c }}$ represent the duration of a successful data transmission and the duration of collisions between data transmissions $\left(T_{\mathrm{csi}, \mathrm{s}}\right.$ and $T_{\mathrm{csi}, \mathrm{c}}$ have similar definition for ECFB frames). Note that Equation 5 can be transformed to Bianchi's model if $\gamma$ equals zero, which means that the ECFB protocol is disabled or the MU-RTS/CTS scheme is in operation.

$$
S=\frac{(1-\gamma) \cdot p_{\mathrm{s}} \cdot N_{\mathrm{f}} \cdot N_{\mathrm{b}} \cdot L}{\gamma \cdot\left(p_{\mathrm{s}} T_{\mathrm{csi}, \mathrm{s}}+p_{\mathrm{c}} T_{\mathrm{csi}, \mathrm{c}}\right)+(1-\gamma) \cdot\left(p_{\mathrm{s}} T_{\mathrm{data}, \mathrm{s}}+p_{\mathrm{c}} T_{\mathrm{data}, \mathrm{c}}\right)+p_{\mathrm{e}} \sigma}
$$

$\tau$, as given in Equation 6, is the sum of the probability that the back-off counter reaches zero, no matter which stage the back-off is in, where $m$ is the maximum back-off stage.

$$
\tau=\frac{2(1-2 p)}{(1-2 p)\left(\mathrm{CW}_{\min }+1\right)+p \mathrm{CW}_{\min }\left(1-(2 p)^{m}\right)}
$$

$p$ is the conditional collision probability for a node that transmits a frame, and in that slot, at least one of the $n-1$ nodes is also transmitting.

$$
p=1-(1-\tau)^{n-1}
$$


$\gamma$ can be derived through a given $T_{2 \text {-CSI-Req value as }}$ follows:

1. The total number of slots in $T_{2 \text {-CSI-Req is }} N_{\text {slots }}=$ $\frac{T_{2 \text {-CSI-Req }}}{E\left[T_{\text {slot }}\right]}$, where $E\left[T_{\text {slot }}\right]$ is the average slot duration (the denominator of Equation 5).

2. The average number of slots in the CSI mode during

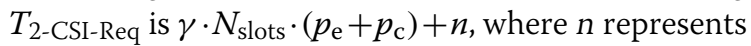
the number of successful ECFB transmissions, one for

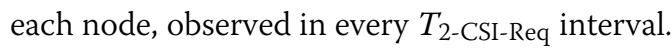

3. Lastly, the probability of ECFB, i.e., the probability that the channel is in the CSI mode, is $\gamma=\frac{\gamma \cdot N_{\text {slots }} \cdot\left(p_{\mathrm{e}}+p_{\mathrm{c}}\right)+n}{N_{\text {slots }}}$, which can be simplified to Equation 8. It shows that the probability of being in the CSI mode can be represented as the number of successful slots in the CSI mode divided by the total number of successful slots in $T_{2 \text {-CSI-Req }}$.

$$
\gamma=\frac{n}{p_{\mathrm{s}} \cdot N_{\text {slots }}}
$$

Equations 5, 6, 7, and 8 present a nonlinear system, which can be resolved using the iterative numerical technique as used in $[10,21]$. If we replace a slot duration of Equation 5 with the corresponding item as shown in Equations 9 and 10 , the saturation throughput $S$ is valid for either the MUBasic scheme or the MU-RTS/CTS scheme.

$$
\left\{\begin{aligned}
T_{\mathrm{data}, \mathrm{c}}^{\mathrm{MU}}= & T_{\mathrm{A}-\mathrm{MPDU}}+\mathrm{SIFS}+T_{\mathrm{B}-\mathrm{ACK}}+\mathrm{AIFS}+\sigma \\
T_{\mathrm{cSi}, \mathrm{c}}^{\mathrm{MU}}= & T_{\mathrm{NDPA}}+\mathrm{SIFS}+T_{\mathrm{NDP}}+\mathrm{SIFS} \\
& +T_{\mathrm{BF}-\mathrm{Feedback}}+\mathrm{AIFS}+\sigma \\
T_{\mathrm{data}, \mathrm{c}}^{\mathrm{MU} / \mathrm{CTS}}= & T_{\mathrm{RTS}}+\mathrm{SIFS}+T_{\mathrm{MU}-\mathrm{CTS}}+\mathrm{AIFS}+\sigma
\end{aligned}\right.
$$

$$
\left\{\begin{aligned}
T_{\text {data,sasic }}^{\mathrm{MU}}=T_{\mathrm{A}-\mathrm{MPDU}}+N_{\mathrm{b}} \cdot\left(\mathrm{SIFS}+T_{\mathrm{B}-\mathrm{ACK}}\right)+\mathrm{AIFS}+\sigma & \\
T_{\mathrm{csi}, \mathrm{s}}^{\mathrm{MU}}= & T_{\mathrm{NDPA}}+T_{\mathrm{NDP}}+(n-1) \cdot T_{\mathrm{BF}-\mathrm{Feedback}} \\
& +(n-2) \cdot T_{\mathrm{Poll}}+(2 n-2) \cdot \mathrm{SIFS}+\mathrm{AIFS}+\sigma \\
T_{\mathrm{data}, \mathrm{s}}^{\mathrm{MU} / \mathrm{CTS}} & =T_{\mathrm{RTS}}+\mathrm{SIFS}+N_{\mathrm{b}} \cdot\left(T_{\mathrm{MU}-\mathrm{CTS}}+\mathrm{SIFS}\right) \\
& +T_{\mathrm{A}-\mathrm{MPDU}}+\mathrm{SIFS}+T_{\mathrm{B}-\mathrm{ACK}}+\mathrm{AIFS}+\sigma
\end{aligned}\right.
$$

The duration of each frame transmission can be calculated as shown in Equation 11 , where $T_{\mathrm{VHT}}(M)=(36+$ $M$. 4) $\mu$ s are the duration of the IEEE 802.11ac PHY preamble (the number of VHT-LTF is proportional to the number of antenna $M) . N_{\mathrm{S}}$ is the number of spatial streams in each beam. $L_{\text {service, }} L_{\text {tail }}$, and $L_{\text {delimiter }}$ are the length of the service field, the tail field, and the MPDU delimiter. $L_{\mathrm{MAC}}$ is a MAC header that will be added to the calculation if it is a data frame. $N_{\text {DBPS }}$ and $T_{\text {symbol }}$ are the number of data bits in a symbol and the symbol duration. $L_{\mathrm{RTS}}$, $L_{\mathrm{MU}-\mathrm{CTS}}$, and $L_{\mathrm{B}-\mathrm{ACK}}$ are the length of RTS, MU-CTS, and
B-ACK, respectively. The detailed calculation of the frame duration can be found in [8].

$$
\left\{\begin{array}{l}
T_{\mathrm{A}-\mathrm{MPDU}}=T_{\mathrm{VHT}}(M)+\left\lceil\frac{L_{\text {service }}+N_{\mathrm{f}} \cdot\left(L_{\mathrm{MAC}}+L+L_{\text {delimiter }}\right)+L_{\text {tail }}}{N_{\mathrm{s}} \cdot N_{\mathrm{DBPS}}}\right\rceil T_{\text {symbol }} \\
T_{\mathrm{RTS}}=T_{\mathrm{VHT}}(M)+\left\lceil\frac{L_{\text {service }}+L_{\mathrm{RTS}}+L_{\text {tail }}}{N_{\mathrm{DBPS}}}\right\rceil T_{\text {symbol }} \\
T_{\mathrm{MU}-\mathrm{CTS}}=T_{\mathrm{VHT}}(M)+\left\lceil\frac{L_{\text {service }}+L_{\mathrm{MU}-\mathrm{CTS}}+L_{\text {tail }}}{N_{\mathrm{DBPS}}}\right\rceil T_{\text {symbol }} \\
T_{\mathrm{B}-\mathrm{ACK}}=T_{\mathrm{VHT}}(M)+\left\lceil\frac{L_{\text {service }}+L_{\mathrm{B}-\mathrm{CAK}}+L_{\text {tail }}}{N_{\mathrm{D} \text { BPS }}}\right\rceil T_{\text {symbol }}
\end{array}\right.
$$

\subsection{Performance results}

The parameters considered for the performance evaluation of MU-Basic and MU-RTS/CTS are shown in Table 4. A simulator has been developed in $\mathrm{C}++$ using the Component Oriented Simulation Toolkit library [22]. Results obtained by the simulations are compared with those of the analytical model.

Figure 8 shows the system throughput against the interval between two CSI requests $\left(T_{2-\mathrm{CSI}-\mathrm{Req}}\right)$. The results clearly show that the system (MU-Basic) obtains a higher throughput if the CSI is less frequently updated. The throughput of the MU-RTS/CTS scheme is not affected by increasing $T_{2-C S I-R e q}$ because the channel sounding process is integrated into the RTS and MU-CTS exchanges.

Note that the results of analytical model and simulations for the MU-RTS/CTS scheme match very well, while there are some gaps for the MU-Basic scheme. It is because, in

\section{Table 4 System parameters}

\begin{tabular}{ll}
\hline Parameters & Values \\
\hline Channel bandwidth & $160 \mathrm{MHz}$ \\
Modulation and coding scheme & $256-\mathrm{QAM}$ with $5 / 6$ \\
Guard interval & $0.8 \mu \mathrm{s}$ \\
Frame length $(L)$ & 20,000 bits \\
MAC header $\left(L_{\text {MAC }}\right)$ & 272 bits \\
MPDU delimiter $\left(L_{\text {delimiter }}\right)$ & 32 bits \\
Service bits $\left(L_{\text {service }}\right)$ & 16 bits \\
Tail bits $\left(L_{\text {tail }}\right)$ & 6 bits \\
RTS & 160 bits \\
MU-CTS & $112+\left(M \cdot N_{\text {dsc }} \cdot 8\right)$ bits \\
B-ACK & $192+\left\lceil N_{f} / 87 \cdot 8\right.$ bits \\
NDPA & $152+n \cdot 16$ bits \\
NDP & $36+M \cdot 4 \mu \mathrm{s}$ \\
VHT compressed beamforming frame & $40+\left(M \cdot N_{\text {dsc }} \cdot 8\right)$ bits \\
Poll & 168 bits \\
Slot time $(\sigma)$ & $9 \mu \mathrm{s}$ \\
SIFS and AlFS & 16 and $34 \mu \mathrm{s}$ \\
CWmin and $C W_{\text {max }}$ & 16 and 1,024 \\
Maximum back-off stage $(m)$ & 6 \\
\hline
\end{tabular}




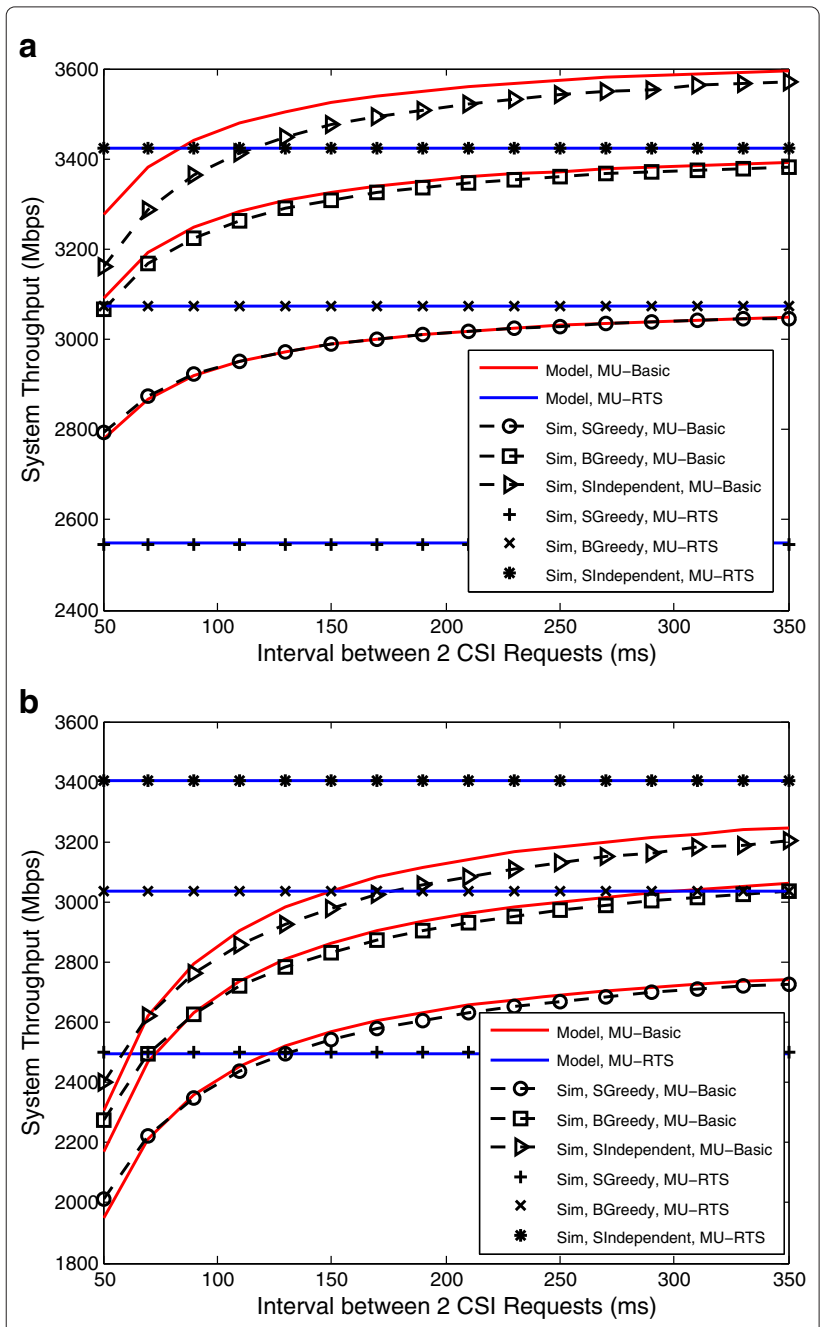

Figure 8 Throughput against $\boldsymbol{T}_{\text {2-CSI-Req }}$ (a) $n=5$. (b) $n=10$.

the MU-Basic scheme, we leave collisions between ECFB frames and data frames out of the analytical model to simplify the calculation; however, these collisions are counted in the simulation to resemble the real scenario. Therefore, the analytical results of MU-Basic are slightly more optimistic than those of simulations. It is also observed that the results (the analytical and simulations) of the Stream-greedy algorithm match better than the other two algorithms, which is because the channel sees a lower percentage of $\gamma$ if the Stream-greedy algorithm is running (the more streams, the shorter data transmission duration and the more data slots in $\left.T_{2 \text {-CSI-Req }}\right)$, therefore reducing the impact of the assumption of the analytical model compared to the two other algorithms.

As shown in Figure 8, the system throughput of MU-Basic increases as the CSI updates less frequently (lower $\gamma$ ). In a slow-mobility scenario (e.g., the maximum Doppler shift is $12 \mathrm{~Hz}$ ), the coherence time of a $5-\mathrm{GHz}$ channel is in the range of 50 to $80 \mathrm{~ms}[23,24]$. Therefore, we set $T_{2 \text {-CSI-Req }}$ to $80 \mathrm{~ms}$ in the following simulations.

Figure 9 shows the throughput against the number of nodes, where $N_{\mathrm{f}}=64, M=8$, and $T_{2 \text {-CSI-Req }}=80$ ms. The results show that the Beam-greedy scheme outperforms the Stream-greedy, although the ideal Streamindependent scheme performs best. The throughput of all schemes decreases as the number of nodes increases. However, the decreasing rate of the MU-Basic scheme is higher than that of the MU-RTS/CTS. The reason is that collisions occur more frequently as the number of nodes increases; however, the collision time of the MU-RTS/CTS scheme is less than that of the MU-Basic scheme as RTS is much shorter than the data frame in length. The other hint from Figure 9 is that the size of the IEEE 802.11ac wireless mesh network could be heavily limited if the MU-Basic scheme is adopted due to the CSI overhead.

Figure 10 shows the throughput against $M$, where $N_{\mathrm{f}}=64, T_{2 \text {-CSI-Req }}=80 \mathrm{~ms}$, and $n=5$ and 10 . The throughput of the Stream-independent scheme always increases with $M$ and outperforms the other schemes. It is because the Stream-independent scheme not only transmits data in parallel but also transmits overheads concurrently. The system throughput of Stream-greedy and Beam-greedy increases in the following ranges: $M=$ 2 to $M=4, M=5$ to $M=6$, and $M=7$ to $M=8$. This is due to the increase of $N_{\mathrm{s}}$ and $N_{\mathrm{b}}$, which either decreases the transmission duration or increases the number of simultaneously transmitted A-MPDUs. The system throughput of Stream-greedy and Beam-greedy slightly decreases in the ranges of $M=4$ to $M=5$ and $M=6$ to $M=7$, which is because $N_{\mathrm{s}}$ and $N_{\mathrm{b}}$ do not increase there, while the CSI overhead increases proportionally to $M$.

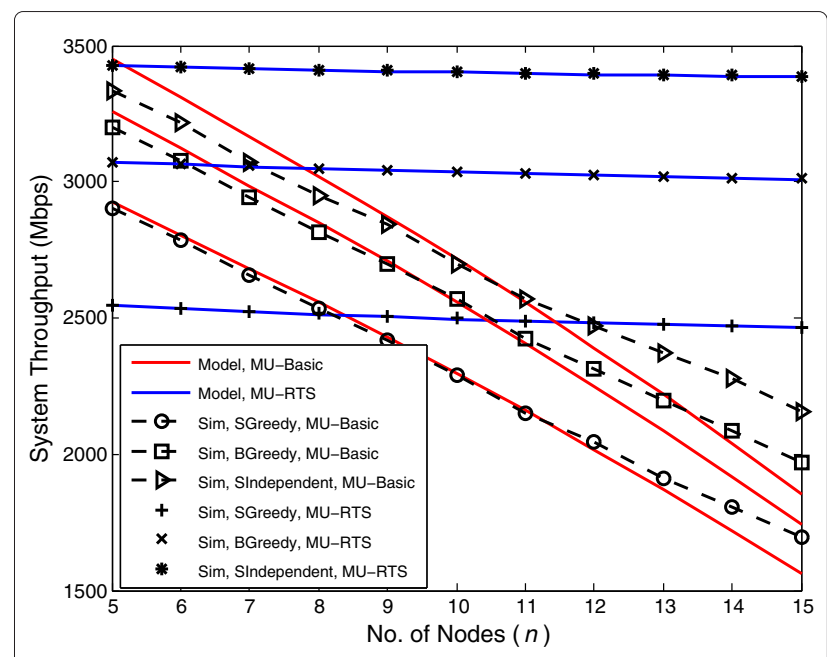

Figure 9 Throughput against $n$. 


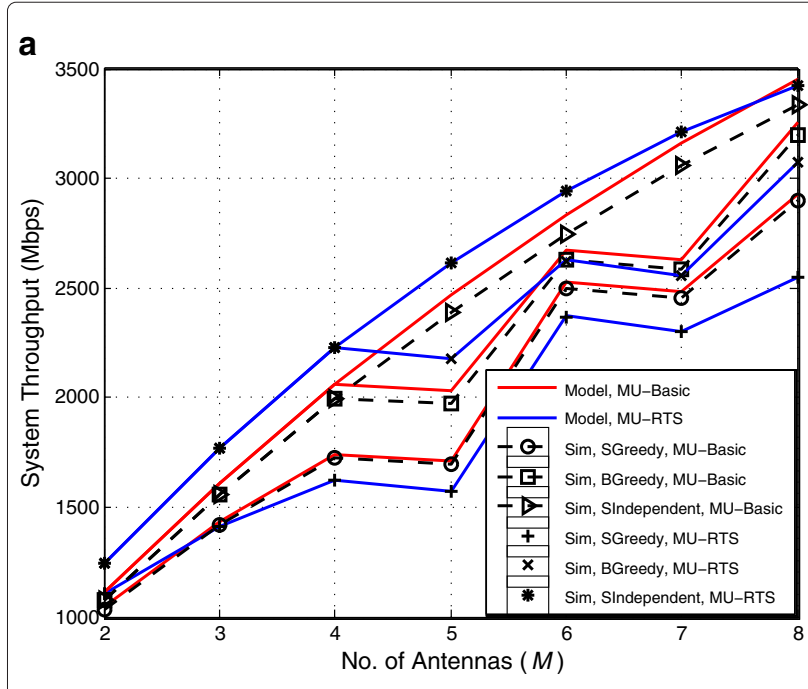

b

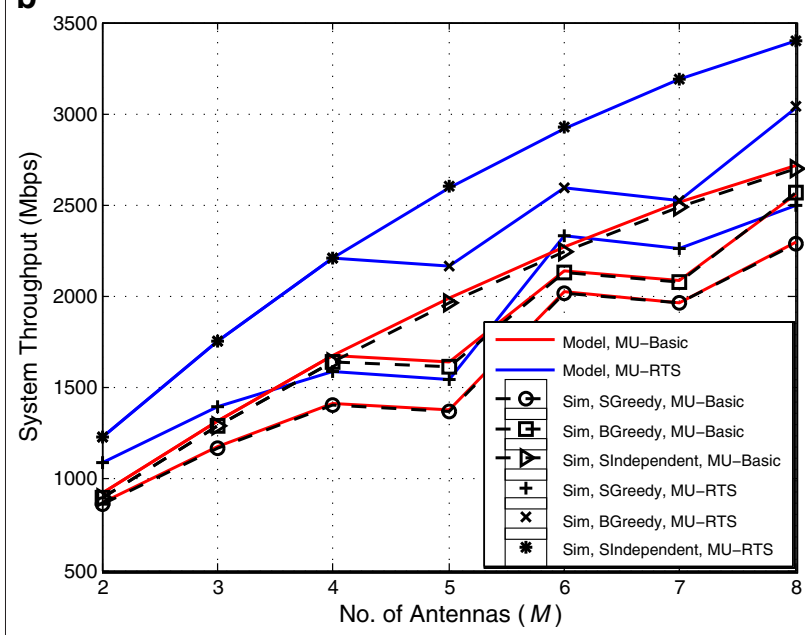

Figure 10 Throughput against $\boldsymbol{M}$. (a) $n=5$. (b) $n=10$.

Figure 11 shows the throughput against $N_{\mathrm{f}}$, where $M=8, T_{2 \text {-CSI-Req }}=80 \mathrm{~ms}$, and $n=5$ and 10 . It clearly shows that the system throughput increases as $N_{\mathrm{f}}$ becomes larger. At the points where $N_{\mathrm{f}}$ is large, the performance of the MU-RTS/CTS scheme exceeds that of the MU-Basic, which is because the RTS and MU-CTS exchanging process is more effective in case of collisions, given that the extra overheads of MURTS/CTS are compensated by the shorter collision duration.

Figure 12 shows the throughput against the channel bandwidth, where $M=8, T_{2 \text {-CSI-Req }}=80 \mathrm{~ms}, N_{\mathrm{f}}=$ $64, n=5$ and 10. It shows that the system throughput increases if a wider channel bandwidth is used; however, the increase is not linear with the channel bandwidth. It is due to the increase of CSI overheads (i.e., the size of the CSI feedback increases with the channel bandwidth), as well as the constant PHY header duration

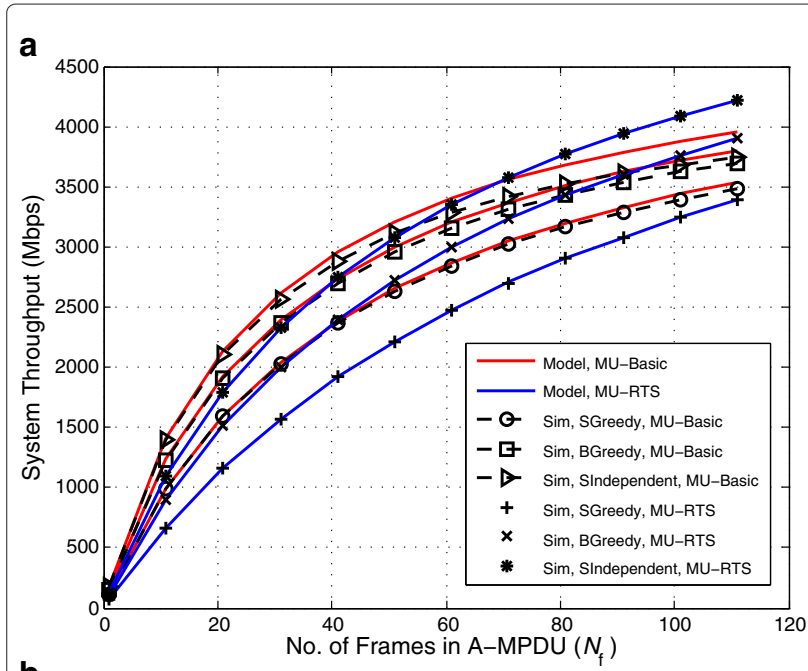

b

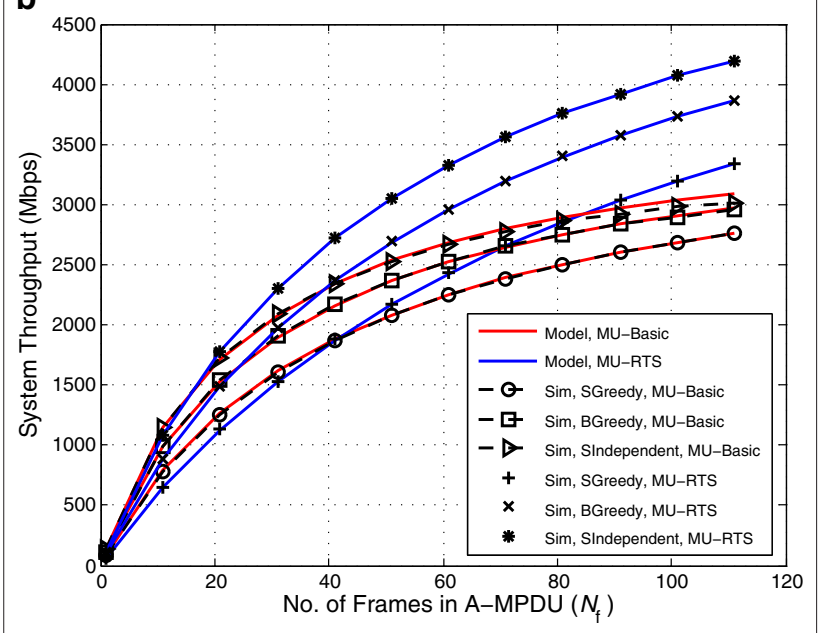

Figure 11 Throughput against $\boldsymbol{N}_{\mathbf{f}} \cdot(\mathbf{a}) n=5$. (b) $n=10$.

(as described in Section 2.2). An effective way to compensate the growth of overheads is to increase $N_{\mathrm{f}}$ together with the bandwidth, which reveals that the system parameters have to be jointly considered to maximize the system performance.

\section{Conclusions}

In this paper, a simple but accurate analytical model is presented for IEEE 802.11ac wireless mesh backhaul networks in saturated conditions. The IEEE 802.11acdefined basic access scheme and proposed MU-RTS/CTS scheme are evaluated and compared through three spatial stream allocation algorithms by analytical model and simulations. The results show that MU-RTS/CTS is more efficient than MU-Basic as the number of nodes and the size of A-MPDU increase. Regarding the spatial stream allocation algorithms, the Beamgreedy algorithm outperforms the Stream-greedy, but the 


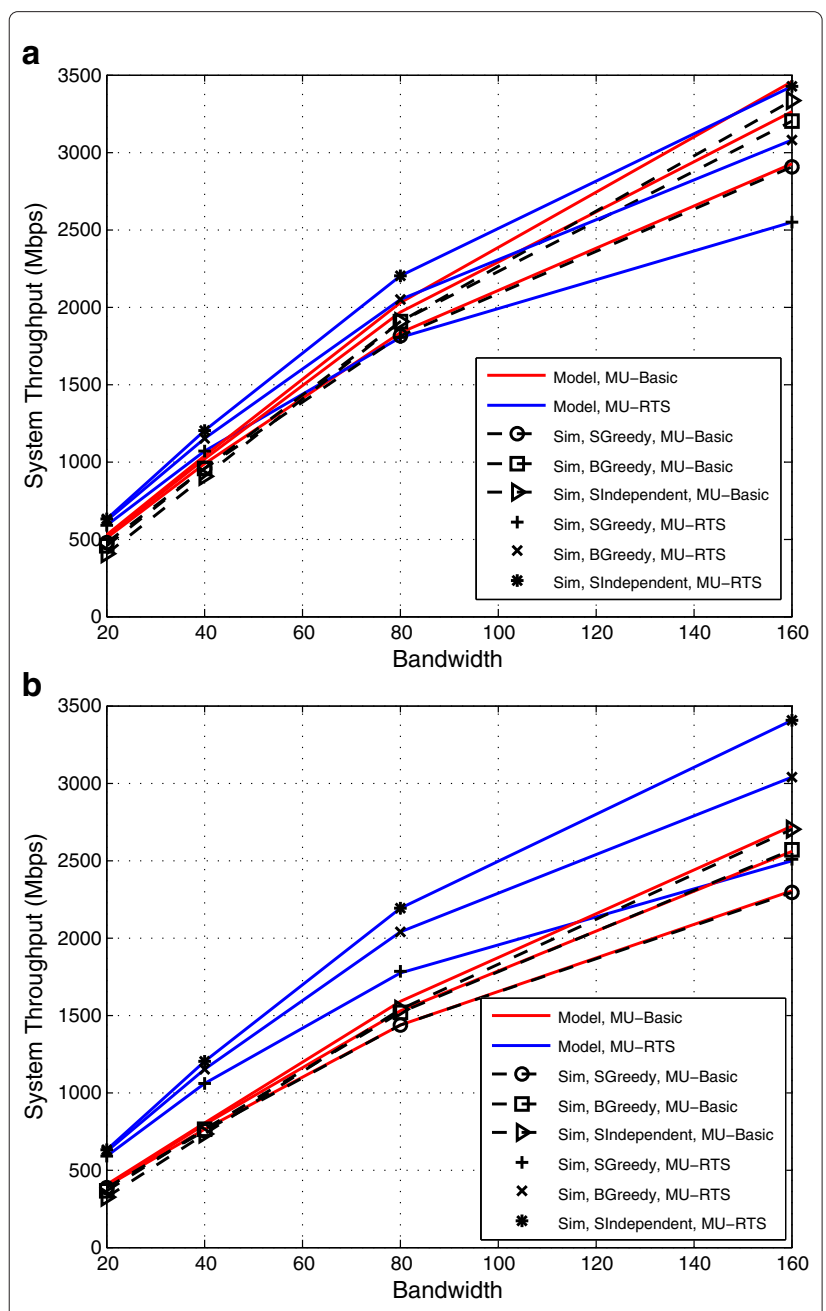

Figure 12 Throughput against bandwidth. (a) $n=5$. (b) $n=10$.

ideal Stream-independent algorithm provides the best performance.

From the presented results, it is clear that the novel PHY and MAC features introduced by IEEE 802.11ac, such as the downlink MU-MIMO, the frame aggregation, and the channel bonding, are able to provide significant performance gains. However, the results also point out the importance of the spatial stream allocation algorithm and the high overheads introduced by the CSI acquisition procedure. The wireless mesh network may not work well in some scenarios where the number of nodes is high and CSI is updated frequently if MU-MIMO transmissions are considered.

Using this paper as a basis, there are several open research challenges that can be considered in the future work:

1. Scheduling scheme. An adaptive scheduling algorithm that is able to jointly consider several important parameters would play a significant role in improving the system performance. As hinted from the above results, these factors include the spatial stream/frame allocation, the number of nodes/antennas, the size of A-MPDU, the channel bandwidth, the queueing state, and the interference conditions. Besides, it also needs to take the following points into account: minimizing the frequency of channel sounding, maximizing the system throughput, and not being unfair to the active nodes.

2. Multi-packet reception (MPR). In this paper, we proposed an MU-RTS/CTS scheme that allows a node to simultaneously transmit frames to multiple nodes. However, MPR (i.e., simultaneous transmissions from multiple nodes to one node), which is able to reduce the collision probability and therefore improve the system performance, has not been considered. Normally, MPR requires synchronization among distributed nodes, which makes the MURTS/CTS handshaking process a better candidate than the MU-Basic to be extended to support MPR.

3. Non-saturated conditions. In non-saturated conditions, the ECFB channel sounding policy should be redesigned to reduce overheads. The easiest option is to make on-demand CSI request to some specified nodes only when the transmitter has frames directed to them, while a more complex option can be a node caching the obtained CSI for a predefined time and only requesting for the CSI updates if the node has frames to send and the cached CSI is outdated.

4. Multi-hop mesh networks. The hidden nodes have to be taken into account in a multi-hop wireless mesh network. In addition, the MAC and routing protocols need to be jointly considered due to the fact that there are multiple destinations in a single MU-MIMO transmission.

\section{Competing interests}

The authors declare that they have no competing interests.

\section{Acknowledgements}

The authors would like to thank the editors and the anonymous reviewers for their valuable feedbacks in improving the paper. This work was partially supported by the Spanish and the Catalan governments, through the projects CISNETS (TEC2012-32354) and AGAUR SGR2009-617, respectively.

Received: 21 January 2013 Accepted: 26 August 2013

Published: 10 September 2013

\section{References}

1. IEEE 802.11-2012, Wireless LAN Medium Access Control and Physical Layer Specifications. IEEE Standards (IEEE, Piscataway, 2012)

2. GR Hiertz, D Denteneer, L Stibor, Y Zang, XP Costa, B Walke, The IEEE 802.11 universe. IEEE Commun. Mag. 48, 62-70 (2010)

3. WG802.11-Wireless LAN Working Group, IEEE P802.11ac Draft 5.0: Enhancements for Very High Throughput for Operation in Bands Below $6 \mathrm{GHz}$. IEEE Ongoing Project (IEEE, Piscataway, 2013) 
4. IEEE 802.11n-2009, Amendment 5: Enhancements for Higher Throughput. IEEE Amendment (IEEE, Piscataway, 2009)

5. G Redieteab, L Cariou, P Christin, J-F Helard, PHY+MAC channel sounding interval analysis for IEEE 802.11ac MU-MIMO, in 2012 International Symposium on Wireless Communication Systems (IEEE, Piscataway, 2012), pp. 1054-1058

6. J Cha, H Jin, BC Jung, DK Sung, Performance comparison of downlink user multiplexing schemes in IEEE 802.11ac: MU-MIMO vs. frame aggregation, in 2012 IEEE Wireless Communications and Networking Conference (IEEE, Piscataway, 2012), pp. 1514-1519

7. EH Ong, J Kneckt, O Alanen, Z Chang, T Huovinen, T Nihtila, IEEE 802.11ac: enhancements for very high throughput WLANs, in 2011 IEEE International Symposium on Personal, Indoor and Mobile Radio Communications (IEEE, Piscataway, 2011), pp. 849-853

8. B Bellalta, J Barcelo, D Staehle, A Vinel, M Oliver, On the performance of packet aggregation in IEEE 802.11ac MU-MIMO WLANs. IEEE Commun. Lett. 16, 1588-1591 (2012)

9. D Nojima, L Lanante, Y Nagao, M Kurosaki, H Ochi, Performance evaluation for multi-user MIMO IEEE 802.11ac wireless LAN system, in 2012 International Conference on Advanced Communication Technology (IEEE, Piscataway, 2012), pp. 804-808

10. G Bianchi, Performance analysis of the IEEE 802.11 distributed coordination function. IEEE J. Selected Areas Commun. 18, 535-547 (2000)

11. H Li, A Attar, VCM Leung, Multi-user medium access control in wireless local area network, in 2010 IEEE Wireless Communications and Networking Conference (IEEE, Piscataway, 2010), pp. 1-6

12. Z Zhang, Y Yang, M Zhao, Enhancing downlink performance in wireless networks by simultaneous multiple packet transmission. IEEE Trans. Comput. 58, 706-718 (2009)

13. PX Zheng, YJ Zhang, SC Liew, Multipacket reception in wireless local area networks, in 2006 IEEE International Conference on Communications (IEEE, Piscataway, 2006), pp. 3670-3675

14. J Zhang, HN Lee, Throughput enhancement with a modified 802.11 MAC protocol with multi-user detection support. AEUE-Int J. Electron. Commun. 62, 365-373 (2008)

15. R de Vegt, IEEE 802.11ac usage model document (2009), https://mentor. ieee.org/802.11/dcn/09/11-09-0161-02-00ac-802-11ac-usage-modeldocument.ppt. Accessed 25 June 2013

16. Cisco, 802.11ac: The Fifth Generation of Wi-Fi. Technical white paper (Cisco, San Jose, 2012)

17. LWard, 802.11ac Technology Introduction. Technical white paper (Rohde \& Schwarz, Munich, 2012)

18. E Perahia, R Stacey, Next Generation Wireless LANs: Throughput, Robustness, and Reliability in $802.11 n$ (Cambridge University Press, Cambridge, 2008)

19. R Liao, B Bellalta, C Cano, M Miquel, DCF/DSDMA: enhanced DCF with SDMA downlink transmissions for WLANs, in 2011 Baltic Congress on Future Internet Communications (IEEE, Piscataway, 2011), pp. 96-102

20. R Liao, B Bellalta, M Miquel, DCF/USDMA: enhanced DCF for uplink SDMA transmissions in WLANs, in 2012 International Wireless Communications and Mobile Computing Conference (IEEE, Piscataway, 2012), pp. 263-268

21. A Kumar, E Altman, D Miorandi, M Goyal, New insights from a fixed-point analysis of single cell IEEE 802.11 WLANs. IEEE/ACM Trans. Netw. 15, 588-601 (2007)

22. G Chen, BK Szymanski, Component Oriented Simulation Toolkit (2004), http://www.ita.cs.rpi.edu/cost.html. Accessed 25 June 2013

23. CS Hwang, JM Cioffi, Opportunistic CSMA/CA for achieving multi-user diversity in wireless LAN. IEEE Trans. Wireless Commun. 8, 2972-2982 (2009)

24. J Kivinen, X Zhao, P Vainikainen, Empirical characterization of wideband indoor radio channel at $5.3 \mathrm{GHz}$. IEEE Trans. Antennas Propagation 49, 1192-1203 (2001)

doi:10.1186/1687-1499-2013-226

Cite this article as: Liao et al.: Performance analysis of IEEE 802.11ac wireless backhaul networks in saturated conditions. EURASIP Journal on Wireless Communications and Networking 2013 2013:226.

\section{Submit your manuscript to a SpringerOpen ${ }^{\circ}$ journal and benefit from:}

- Convenient online submission

- Rigorous peer review

- Immediate publication on acceptance

- Open access: articles freely available online

- High visibility within the field

- Retaining the copyright to your article

Submit your next manuscript at $\gg$ springeropen.com 